\title{
FUNCTIONAL UNIFORMITIES
}

\author{
S. B. MYERS
}

This paper is concerned with uniform structures in the sense of André Weil [4]. ${ }^{1}$ In his proof that every Hausdorff uniform space is completely regular, ${ }^{2}$ Weil obtained a result which may be phrased as follows.

WeIL'S THEOREM. If $V$ is a uniformity (uniform structure) on the completely regular space $X$, given an "entourage" $V_{\alpha}$ there exists a uniformly equicontinuous ${ }^{3}$ set $F$ of bounded real functions on $X$ and $a \delta>0$ such that corresponding to each $P \in X$ there is an $f_{P} \in F$ with the property that $Q \in V_{\alpha}(P)$ if $\left|f_{P}(P)-f_{P}(Q)\right|<\delta$.

This result suggests that every uniformity on $X$ can be defined by means of a family of sets of real bounded continuous functions, and that important uniformities on $X$ (such as precompact [1] ones) are definable by means of "nice" sets of such functions. These and related problems are investigated here.

A family $\mathcal{F}$ of sets of real functions will be said to separate a topological space $X$ if for each $P \in X$ and neighborhood $N(P)$ there exists an $F \in \mathcal{F}$ and a $\delta>0$ such that if $|f(Q)-f(P)|<\delta$ for all $f \in F$, then $Q \in N(P)$.

Let $X$ be a completely regular space, let $\mathcal{F}$ be a family of sets of real continuous functions on $X$ with the following two properties:

(a) $\mathcal{F}$ separates $X$.

(b) Each $F \in \mathcal{F}$ is equicontinuous.

For each $F \in \mathcal{F}$ and real number $\delta>0$, let $V_{\alpha}=\{P, Q \in X|| f(P)-f(Q) \mid$ $<\delta$ for all $f \in F\}$. Then these symmetric "entourages" $V_{\alpha}$ satisfy the axioms for a uniformity on $X$. First, $\bigcap_{\alpha} V_{\alpha}=\Delta$, the diagonal in $X^{2}$; for if $(P, Q) \in V_{\alpha}$ for every $\alpha$, it follows that $f(P)=f(Q)$ for every

Presented to the Society, September 1, 1949; received by the editors December 16, 1949.

${ }^{1}$.Numbers in brackets refer to the list of references at the end of the paper.

${ }^{2}$ A completely regular space is a Hausdorff space with the property that given any point $x \in X$ and closed set $K \subset X$ not containing $x$, there exists a real continuous function $f$ such that $f(x)=1, f(y)=0$ for all $y \in K$.

${ }^{3}$ A set $F$ of real functions on a topological space $X$ is equicontinuous at $P \in X$ if given $\epsilon>0$ there exists a neighborhood $N(P)$ such that if $Q \in N(P)$, then $|f(Q)-f(P)|$ $<\in$ for all $f \in F . F$ is equicontinuous if it is equicontinuous at every $P \in X$. If $X$ is a uniform space, $F$ is uniformly equicontinuous if given $\epsilon>0$ there exists an entourage $V_{\alpha}$ such that if $(P, Q) \in V_{\alpha}$, then $|f(Q)-f(P)|<\epsilon$ for all $f \in F$. Note that if the members of an equicontinuous $F$ are uniformly continuous, it does not necessarily follow that $F$ is uniformly equicontinuous. 
$f \in F \in \mathcal{F}$, and by (a) this implies that $Q$ is contained in every neighborhood of $P$, which means that $Q=P$. Second, given $\alpha, \beta$ there exists $\gamma$ such that $V_{\gamma} \subset V_{\alpha} \cap V_{\beta}$; for by (b) there exist open sets $O$ and $O^{\prime}$ in $X^{2}$, both containing $\Delta$, and respectively contained in $V_{\alpha}$ and $V_{\beta}$, hence $O \cap O^{\prime} \subset V_{\alpha} \cap V_{\beta}$; but by (a), there is a $\gamma$ such that $V_{\gamma} \subset O \cap O^{\prime}$. Third, given $\alpha$ there is a $\beta$ such that $V_{\beta} V_{\beta} \subset V_{\alpha}$; for if $\alpha=(F, \delta)$, we need only take $\beta=(F, \delta / 2)$.

This uniformity on $X$ is compatible with the topology of $X$, for it induces a topology which by (a) is as fine as that of $X$, and by (b) is at most as fine as that of $X^{4}$

Such a uniformity $V$, that is, any uniformity thus determined by a separating family of equicontinuous sets of real functions on $X$, will be called a functional uniformity on $X$. If all the members of every $F \in \mathcal{F}$ are bounded, we call $V$ a bounded functional uniformity on $X$.

THEOREM 1. Every uniformity $V$ on a completely regular space $X$ is isomorphic to a bounded functional uniformity.

Proof. Let $\mathcal{F}$ be the family of all uniformly equicontinuous sets of real bounded functions on $V$. By Weil's Theorem, the functional uniformity determined by $\mathcal{F}$ is at least as fine as $V$; the uniform equicontinuity of the members of $\mathcal{F}$ implies that $V$ is at least as fine as the functional uniformity determined by $\mathcal{F}$ :

COROLLARY. The finest uniformity on a completely regular space is the bounded functional uniformity determined by the family of all equicontinuous sets of real bounded functions.

We note in passing the following theorem:

Theorem 2. A completely regular space $X$ is metrizable if and only if it possesses a separating family consisting of a single equicontinuous set of real bounded functions.

Proof. If there exists such a set $F$, let $V$ be the functional uniformity determined by it, so that $V_{\delta}=\{P, Q \in X|| f(Q)-f(P) \mid<\delta$ for all $f \in F\} . V$ is clearly isomorphic to a first countable uniformity, hence [4] to a metric uniformity. Conversely, if $X$ is metrizable, it possesses a bounded metric $\rho(P, Q)$. For a fixed $P, \rho(P, Q)$ defines a bounded continuous function on $X$. The set of functions obtained by

${ }^{4}$ The following interpretation of (a) and (b) was pointed out by the referee. Let $G$ be a family of subsets of $R^{X}$ ( $R=$ reals), for each $G \in G$ let $R^{G}$ be provided with its

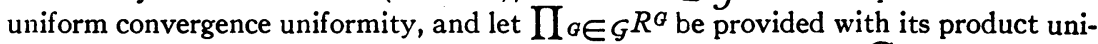
formity. Then if $\mathcal{F}$ is the family of all finite unions of members of $G$, (a) and (b) are necessary and sufficient that the natural mapping $X \rightarrow \prod_{a \in G} R^{G}$ be a homeomorphism. 
letting $P$ range over $X$ is equicontinuous (by the triangle inequality); it separates $X$, because if $P \in X$ and $N(P)$ is a neighborhood of $P$, by taking $\delta$ so that the sphere neighborhood of $P$ of radius $\delta$ lies in $N(P)$, we see that if $|\rho(P, Q)-\rho(P, P)|<\delta$, then $Q \in N(P)$.

If a separating family $\mathcal{F}$ on a completely regular space $X$ consists of finite sets only, the functional uniformity it determines will be called a weak functional uniformity. If in addition all the members of each $F \in \mathcal{F}$ are bounded, it will be called a weak bounded functional uniformity.

Lemma 1. Let $V$ be a uniformity on a completely regular $X$. Suppose an "entourage" $V_{\alpha}$ has the property that there exists $a \delta>0$ and a finite (or infinite of cardinal number $\mu$ ) set $F$ of real bounded continuous functions such that for each $P \in X$ there is a finite set $f_{1}, \cdots, f_{m} \in F$ with the property that $\left|f_{i}(Q)-f_{i}(P)\right|<\delta$ for $i=1, \cdots, m$ implies $Q$ $\in V_{\alpha}(P)$. Then there exists a finite (or infinite of cardinal number $\mu$ ) set of points $E \subset X$ such that $\bigcup_{P \in E} V_{\alpha}(P)=X$.

Proof. First we prove this in the finite case. Suppose $(P, Q)$ $\in V_{\alpha}$ if $|f(Q)-f(P)|<\delta$ for all $f \in F$ (where $F$ is finite). Let $a$ $=\sup _{x \in X, f \in F} f(x), b=\inf _{x \in X, f \in F} f(x)$. Let the closed interval $(a, b)$ be divided into a finite number, say $r$, of closed subintervals $I_{1}, \cdots, I_{r}$ each of length less than $\delta$. Let $p_{1}, \cdots, p_{m}$ be any ordered set of $m$ positive integers (not necessarily distinct) each less than or equal to $r$, and let $E_{p_{1}}, \cdots, p_{m}=\left\{P \in X \mid f_{j}(P) \in I_{p_{j}}\right.$ for $\left.j=1, \cdots, m\right\}$. From each such set $E_{p_{1}}, \cdots, p_{m}$ which is not empty choose a point, and order the resulting finite set $P_{1}, \cdots, P_{n}\left(n\right.$ is at most $\left.r^{m}\right)$. Let $P \in X$; there is clearly an $i$ such that $\left|f_{j}(P)-f_{j}\left(P_{i}\right)\right|<\delta$ for $j=1, \cdots, m$. Hence $\left(P, P_{i}\right) \in V_{\alpha}$. Thus $\bigcup_{i=1}^{n} V_{\alpha}\left(P_{i}\right)=X$.

To complete the proof of the lemma, suppose $V_{\alpha}$ has the property that for each $P \in X$ there is a finite set $F_{P} \subset F$ such that $|f(Q)-f(P)|$ $<\delta$ for all $f \in F_{P}$ implies $Q \in V_{\alpha}(P)$. For any finite set $\bar{F} \subset F$, let $E_{\bar{F}}=\left\{P \in X \mid F_{P} \equiv \bar{F}\right\}$. The set $E_{\bar{F}}$ is a uniform space with the property that if $P, Q \in E_{\bar{F}}$ and $|f(Q)-f(P)|<\delta$ for all $f \in \bar{F}$, then $Q$ $\in V_{\alpha}(\mathrm{P}) \cap E_{\bar{F}}$. Hence, by the proof just given for the finite case, there exists a finite set $[P]_{\bar{F}} \in E_{\bar{F}}$ such that $\bigcup_{P \in[P] \bar{F}} V_{\alpha}(P) \cap E_{\bar{F}}=E_{\bar{F}}$. Since the set of finite subsets $\bar{F}$ of $F$ has the same cardinal number as $F$, the set of sets $E_{\bar{P}}$ has that same cardinal number. Furthermore, $\bigcup_{\bar{P}} E_{\bar{p}}=X$. Hence the set $E=\bigcup_{\bar{P}}[P]_{\bar{F}}$ has the property $X$ $=\bigcup_{P \in E} V_{\alpha}(P)$.

Lemma 2. Let $V$ be a uniformity on a completely regular space $X$. Suppose for every $\alpha$ there exists a subset $E$ of $X$ which is finite (or infinite of cardinal number $\mu$ ) such that $\bigcup_{P \in E} V_{\alpha}(P)=X$. Then for each $\alpha$ 
there exists $a \delta>0$ and a finite set $F$ (or an infinite set $F$ of cardinal number $\mu$ ) of real bounded functions which is uniformly equicontinuous and which has the property that for each $P \in X$ there is an $f_{P} \in F$ such that if $\left|f_{P}(Q)-f_{P}(P)\right|<\delta$, then $Q \in V_{\alpha}(P)$.

Proof. Let $\alpha$ be given. Choose $\beta$ so that $V_{\beta} V_{\beta} \subset V_{\alpha}$. According to Weil's Theorem, there is a $\delta>0$ and a uniformly equicontinuous set $F^{\prime}$ of real bounded functions such that given $P \in X$ there is an $f_{P} \in F^{\prime}$ with the property that $\left|f_{P}(Q)-f_{P}(P)\right|<2 \delta$ implies $Q \in V_{\beta}(P)$. Let $\gamma$ be such that $V_{\gamma} \subset V_{\beta}$, and $(P, Q) \in V_{\gamma}$ implies $\left|f_{P}(Q)-f_{P}(P)\right|<\delta$; this is possible by the uniform equicontinuity of $F$. Let $E \subset X$ be a finite set (or a set of cardinal number $\mu$ ) such that $U_{P \in E} V_{\gamma}(P)=X$. Then the set $F$ of functions given by $f_{P}$ as $P$ ranges over $E$ has the property required. For let $P \in X$, and let $\bar{P} \in E$ be such that $P$ $\in V_{\gamma}(\bar{P})$. Then $\left|f_{\bar{P}}(P)-f_{\bar{P}}(\bar{P})\right|<\delta$. If $\left|f_{\bar{P}}(Q)-f_{\bar{P}}(P)\right|<\delta$, it follows that $\left|f_{\bar{P}}(Q)-f_{\bar{P}}(\bar{P})\right|<2 \delta$. Hence $Q \in V_{\beta}(\bar{P})$. But since $(\bar{P}, P) \in V_{\beta}$, it follows that $Q \in V_{\alpha}(P)$.

THEOREM 3. The following conditions on a uniformity $V$ on a completely regular space $X$ are equivalent.

I. For each $\alpha$, there exists a finite set (or an infinite set of cardinal number $\mu) E \subset X$ such that $\bigcup_{P \in E} V_{\alpha}(P)=X$.

II. For each $\alpha$, there exists $a \delta>0$ and a uniformly equicontinuous set of real bounded functions which is finite (or infinite of cardinal number $\mu$ ) such that for each $P \in X$ there is a finite set $F_{P} \subset F$ such that $Q \in V_{\alpha}(P)$ whenever $|f(Q)-f(P)|<\delta$ for all $f \in F_{P}$.

Proof. By Lemma 2, I $\rightarrow$ II. By Lemma 1, II $\rightarrow$ I.

THEOREM 4. The precompact uniformities are identical with the weak bounded functional uniformities; more precisely, every precompact uniformity is isomorphic to a weak bounded functional uniformity, and every weak bounded functional uniformity is precompact.

PROOF. If $V$ is precompact, it satisfies condition I of Theorem 3 in the finite case [1], hence condition II. Therefore the weak bounded functional uniformity determined on $X$ by the family $\mathcal{F}$ of all finite sets of real bounded uniformly continuous functions on $V$ is at least as fine as $V$; it is not finer than $V$ since every finite set of uniformly continuous functions is uniformly equicontinuous. Conversely, if $V$ is a weak bounded functional uniformity, it satisfies condition II of Theorem 3 in the finite case, hence condition $I$ in the finite case. Hence [1] $V$ is precompact.

Doss [2] has shown that a completely regular space has the prop- 
erty that every real continuous function attains its maximum (or equivalently every real continuous function is bounded) if and only if every uniformity on $V$ is precompact; Hewitt [3] calls such a space pseudo-compact. Now if a uniformity on a completely regular space $X$ is precompact, so is every less fine uniformity; hence a completely regular $X$ is pseudo-compact if and only if the finest uniformity on $X$ is precompact. Using the corollary to Theorem 1, and Theorem 4, we obtain the following theorem.

Theorem 5. A completely regular space $X$ is pseudo-compact if and only if corresponding to every equicontinuous set $F$ of real bounded functions on $X$ and $\delta>0$, there exists a finite set $f_{1}, \cdots, f_{m}$ of real bounded continuous functions and $a \delta^{\prime}>0$ such that $\left|f_{i}(Q)-f_{i}(P)\right|<\delta^{\prime}$ for $i=1, \cdots, m$ implies $|f(Q)-f(P)|<\delta$ for all $f \in F$.

Another consequence of Theorem 4 is the following.

THEOREM 6. Given any uniformity $V$ on a completely regular space $X$, there exists a precompact uniformity $V^{\prime}$ on $X$ which is at most as fine as $V$ and has the same set of real bounded uniformly continuous functions as $V$. The uniform completion of $V^{\prime}$ furnishes a compactification $\bar{X}$ of $X$ such that the only real continuous functions on $X$ which can be extended to $\bar{X}$ are those which are uniformly continuous on $V$.

For the weak bounded functional uniformity $V^{\prime}$ on $X$ determined by the family $\mathcal{F}$ of all finite sets of real bounded uniformly continuous functions on $V$ is at most as fine as $V$, since the family $\mathcal{F}$ is contained in the family of all uniformly equicontinuous sets of real bounded functions on $V$, and the uniformity determined by the latter is isomorphic to $V$. By definition of $V^{\prime}$, every real bounded function uniformly continuous on $V$ is uniformly continuous on $V^{\prime}$, and the converse holds because $V^{\prime}$ is at most as fine as $V$.

THEOREM 7. If $V$ is a weak functional uniformity on a completely regular $X$, for each $\alpha$ there exists a countable set $E \subset X$ such that $\bigcup_{P \in E} V_{\alpha}(P)$ $=X$.

Proof. Let $\mathcal{F}$ be the family of finite sets of continuous functions on $X$ which determines $V$. Given $\alpha$, let $F, \delta$ be such that $V_{\alpha}=\{P, Q \in X|| f(P)-f(Q) \mid<\delta$ for all $f \in F\}$. For each positive integer $n$, let $E_{n}=\{P \in X \mid-n \leqq f(P) \leqq n$ for all $f \in F\}$. Each $E_{n}$ is a uniform space satisfying the hypotheses of Lemma 1 with the given $\alpha, \delta, F$. Hence a finite number of $V_{\alpha}(P)\left(P \in E_{n}\right)$ covers $E_{n}$. But $X=\bigcup_{n} E_{n}$. Hence a countable number of $V_{\alpha}(P)(P \in X)$ covers $X$.

It follows from Theorems 7 and 3 that every weak functional 
uniformity on $X$ satisfies condition II of Theorem 3 with $\mu=\boldsymbol{N}_{0}$. This could also be proved directly as follows. Let $V_{\alpha}=\left\{P, Q \in X \mid f_{i}(Q)\right.$ $-f_{i}(P) \mid<\delta$ for $\left.i=1, \cdots, m\right\}$. Let $F_{i}=f_{i} /\left(\left|f_{i}\right|+1\right)$ and consider the countable set of bounded functions $n F_{i}$ ( $n$ ranges over the positive integers). Let $P \in X$. Since $f_{i}=F_{i} /\left(1-\left|F_{i}\right|\right)$, it is clear that there exists $\delta_{i}^{\prime}$ such that if $\left|F_{i}(Q)-F_{i}(P)\right|<\delta_{i}^{\prime}$, then $\left|f_{i}(Q)-f_{i}(P)\right|$ $<\delta$. Choosing $n$ so large that $n \delta_{i}^{\prime}>\delta$ for all $i=1, \cdots, m$, we see that if $\left|n F_{i}(Q)-n F_{i}(P)\right|<\delta$, then $\left|f_{i}(Q)-f_{i}(P)\right|<\delta$.

\section{REFERENCES}

1. N. Bourbaki, Eléments de mathématique II, vol. III, Topologie generale, Paris, 1940.

2. R. Doss, On continuous functions in uniform spaces, Ann. of Math. vol. 48 (1947) p. 843.

3. E. Hewitt, Rings of real-valued continuous functions. I, Trans. Amer. Math. Soc. vol. 64 (1948) p. 67.

4. A. Weil, Sur les espaces d̀ structure uniforme et sur la topologie générale, Actualités Scientifiques et Industrielles, no. 551, Paris, 1937.

UNIVERSITY OF MichigAN 